\title{
Reengineering MANET Routing using Ant Colony Optimization: Modelling and Performance Study
}

\author{
Bright Selorm K. Anibrika \\ Comp. Science Department, \\ Koforidua Technical University \\ Box KF 981, KTU, Koforidua
}

\author{
Michael Asante \\ Computer Science Dept., \\ Kwame Nkrumah Univ. of \\ Science and Technology \\ Private Mail Bag, Kumasi
}

\author{
Benjamin Hayfron-Acquah \\ Computer Science Dept., \\ Kwame Nkrumah Univ. of \\ Science and Technology \\ Private Mail Bag, Kumasi
}

\author{
Ebenezer K. Gavua \\ Comp. Science Department, Koforidua Technical University \\ Box KF 981, KTU, Koforidua
}

\begin{abstract}
A wireless network topology that comprise heterogeneous routing paths without the supported of any preliminary network infrastructure is known as a Mobile Ad Hoc Network. Therefore, the nodes have the capacity to forward packets to nodes. Furthermore, MANET properties, namely; dynamic topology, nodal mobility, provide a large degree of freedom and the ability to self-organize give them an urge over other network architectures and topologies. For that matter, designing and developing secure routing algorithms becomes a daunting task for the researcher. To this end, this paper seek to compare preexisting and proposed routing algorithm for MANET based on the mechanism of the ant system, hence Ant Colony Optimization frame would be adopted. It is notable that MANET bandwidth, radio propagation, energy supply, etc. Different MAC protocols have proposed for adhoc networks. In this research, a new algorithm based on the Ant Colony Optimization algorithm framework is proposed. The proposed Ant Colony Optimization algorithm known as Optimized Multicast Routing Algorithm was implemented with the aid of MANET simulation in Mathlab and a performance comparison was carried out. The study compared, the propriety protocol AODV, with the proposed algorithm. It was observed the proposed protocol outperformed AODV under the quantitative metrics used.
\end{abstract}

\section{General Terms}

Routing Protocols, Energy-Efficiency, Wireless Routing. Ant Colony Optimization.

\section{Keywords}

Ants, Algorithm, Nodes, Pheromone, Routing.

\section{INTRODUCTION}

Applications such as emergency disaster relief to networking laptops in a conference room rely on Mobile Ad Hoc Networks for operability. Despite MANET challenges, several optimization models to address these routing algorithms were proposed in the past, each (routing algorithm) is primarily responsible for determining the minimum routes to reach the destination from the source as described in (Perkins and Bhagwat, 1994; Jacquet et al., 2001). Also, extensive research were out in the area of energy-efficient routing algorithms. Find below the conceptual view of using ACO algorithms in modelling routing problems (Royer et al., 2001; Patel and Kamboj, 2015), see Fig. 1.

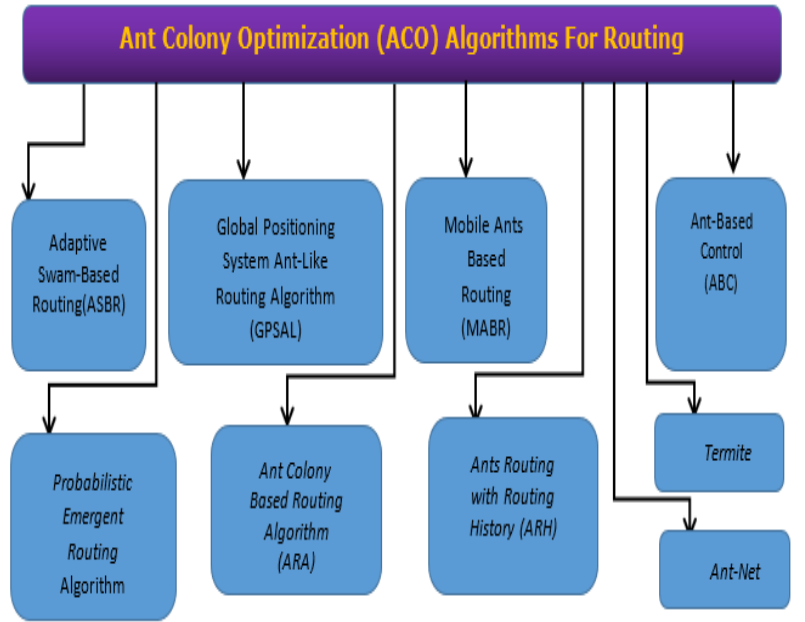

Fig. 1: A Pictorial Representation of Ant Colony Optimization Algorithms (Anibrika et al., 2020; Dorigo et al., 2006)

\subsection{Problem Statement}

Mobile devices engaged in MANET communication have the potential to connect to wireless networks. The tendency of such mobile devices to connect to wireless networks is termed as Mobile Ad-hoc NETworks (Jha, 2010; Kumar et al, 2013). These networks are infrastructureless in nature. Because they lack infrastructure as their "building block", a MANET could be deployed with lower power costs. Due to the nature MANET, routing in such networks are bedeviled with numerous challenges. These challenges are worsened the unpredictable nature of nodal communication especially in finding the shortest routes from source to destination. Several algorithm were proposed to address such challenges. One class of such algorithms is termed Ant Colony Optimization (ACO) algorithms, (Dorigo et al., 2006). ACOs aside their ability to address routing challenges in MANET are also energy-efficient as they turn conserve the energy of the nodes engaged in routing within MANET. The ant algorithm is iterative, probabilistic meta-heuristic which are intended to provide optimal solutions to combinatorial optimization problems most importantly in MANETs (Tonk, 2012). In searching for food, ants exchange information through pheromone deposits, which is a mark of their respective route from their nests to a food source and that also attracts more ants to access the route. In some cases, a dynamic problem 
might occur, demanding a change over time where the algorithm need to monitor by keeping track of the critical updates and consistently optimize the algorithm so as to generate efficient solutions. The ant algorithm has several striking characteristics such as its dynamism, robustness, adaption and its decentralized nature during node processing (Tonk, 2012; Singh, 2013). These characteristics for dynamic routing in modern wireless networks as in the case of MANETs. To this end, this paper investigates Ant Colony Optimization algorithms and their application to MANETs as well as design a new multicast MANET routing algorithm that is energy-efficient. Finally, a comparative study through simulation would be carried out between AODV and the proposed algorithm for MANET.

\section{LITERATURE REVIEW}

\subsection{Ant-Based Control Algorithms}

Research work in the past had focused on Swarm Intelligent routing algorithms as described (Dorigo et al., 2006). Initially, this algorithm was applied to circuit switched networks, like the telephony network. However, the ABC algorithm was later modeled analytically in (Singh, 2013). Again, a critical component of this design $(\mathrm{ABC})$ is the congestion-free mechanism introduced stop calls from dropping intermittently. In (Subramani A and Krishnan A, 2011), the network topology and its routing updates are theoretically modeled by means of a graph. Furthermore, each available node is indicated by a total capacity $C_{i}$, a spare capacity $S_{i}$ and an equally important routing table $R i$. A link in the form of $(i, j)$ exemplifies, a vector quantity of unique pheromone values $T_{j}, d$, which implies that, each destination $d$, represents the tendency of a node traversing from $i$ to $j$ (Nand and Shama, 2011). For this reasoning, the probabilistic values in the routing table are rationalized as the ant traverses the node. This is influenced by the pheromone trails and its intensities. This is furthermore explained in Fig 2.0 below.

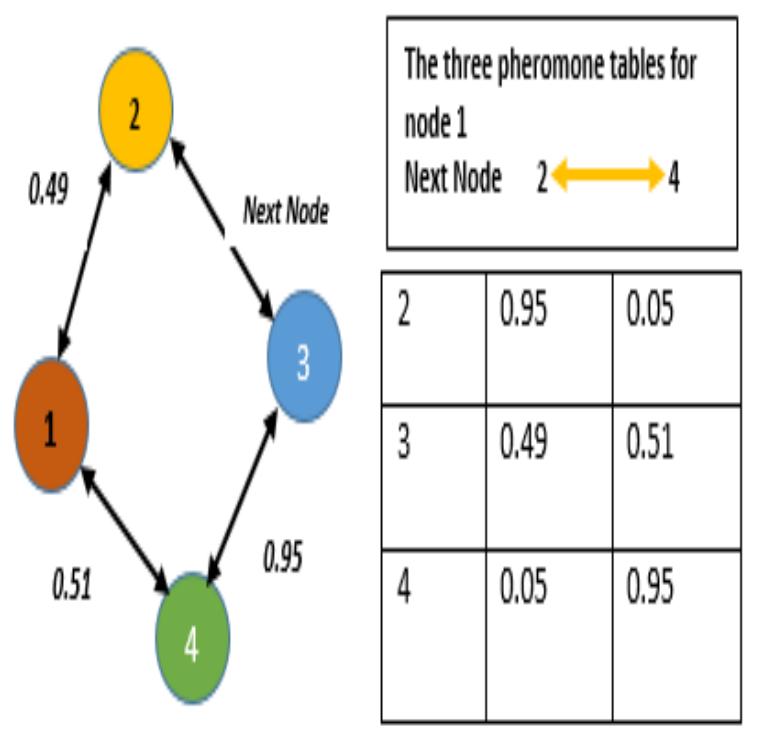

Fig. 2. Pheromone Table Update (Anibrika et al., 2020)

\subsection{Ant Based Control Routing with Probabilistic Update}

During the routing mechanism, routing tables get replaced by pheromone table entries. Also, each node within Ant Based Control network keeps a copy of the pheromone table for every other node that form an integral part of the network routing. Furthermore, each pheromone table has a routing entry for each neighbor node, demonstrating the probability of using that neighbor's node as the next hop. The choice of a node choice is probabilistic while route creation is deterministic Dorigo et al., 2006 ; Nand and Shama, 2011). A further explanation in Fig. 3 is found below:

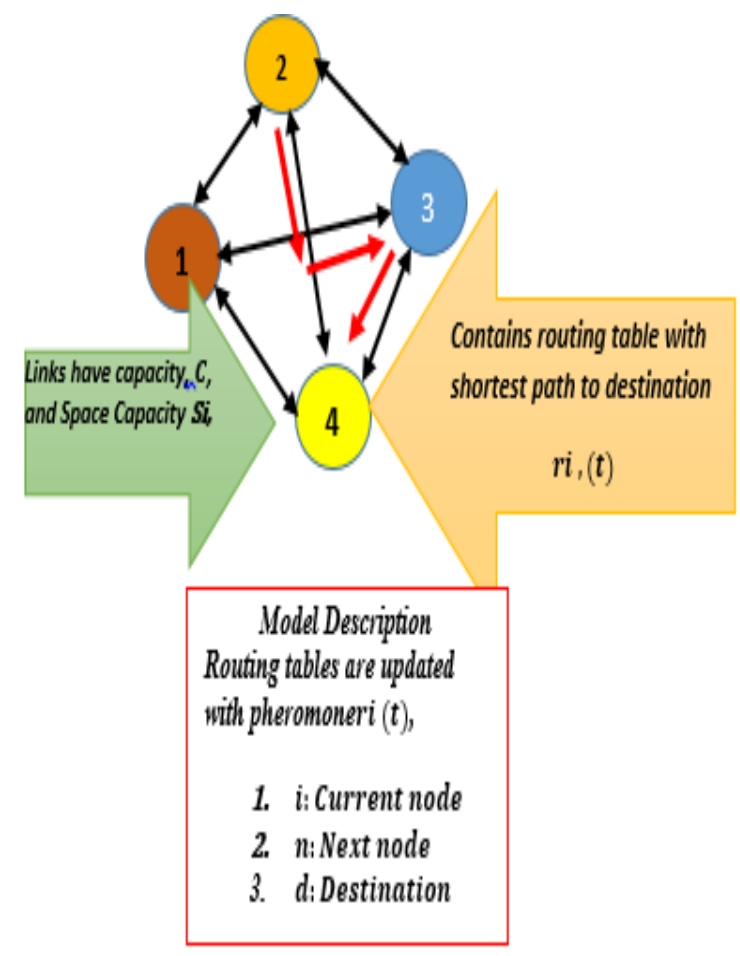

Fig. 3. ABC model for pheromone update

\subsection{AntNet Algorithm}

According to (DiCaro and Dorigo, 2006; Shukla et al., 2013), it is important to develop an improved algorithm influenced by pheromone updates whereby an ant colony move systematically in a food search process. It involves, the use of stochastic decision making with local policy, which is once an ant develops a possible solution to the problem. The ants evaluate possibilities of the solution and leaves pheromone trails (memory buffer) on the path about the connection the ants established. Finally, this specialized information would now be used to determine the forward-bias movements of future ants (Rafiq et al., 2013). In the same vein (Atsushi Iwata et al., 1999) described the traditional ACO algorithm employed using a fixed amount of pheromone as means of updating the pheromone table. (Atsushi Iwata et al., 1999), however developed an adaptive adjustment strategy for the pheromone introduced, making a relatively uniform pheromone distribution based on probabilities. This strategy can deal effectively with the confusion of expanding the ants' search and finding optimal solution algorithms, for finding the local optimal solution (Dorigo, 2006).

\subsection{Updating Routing Tables}

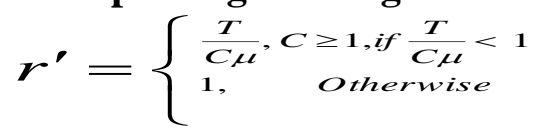

The trip time of the ant is represented by $T$. The scaling factor is denoted by $C$, where 2 is the default value. The mean and variance also denote the round trip time. Furthermore, $=\sigma / \mu$ , which is a ratio of the variance to the mean, denotes a 
quantitative measurement (Dorigo, 2006; Anibrika et al., 2020).

\subsection{Mobile Ad-Hoc Networks}

The topographic structure is completely infrastructureless. This phenomenon has created numerous challenges for MANET research, since the ultimate aim of the routing mechanism is unclear due to several challenges such as limited bandwidth, power, and latency (Atsushi Iwata et al., 1999; M. Bergamo; Jha, 2010; Chlamtac et al., 2003). The diagram in Fig. 4 below, explains further.

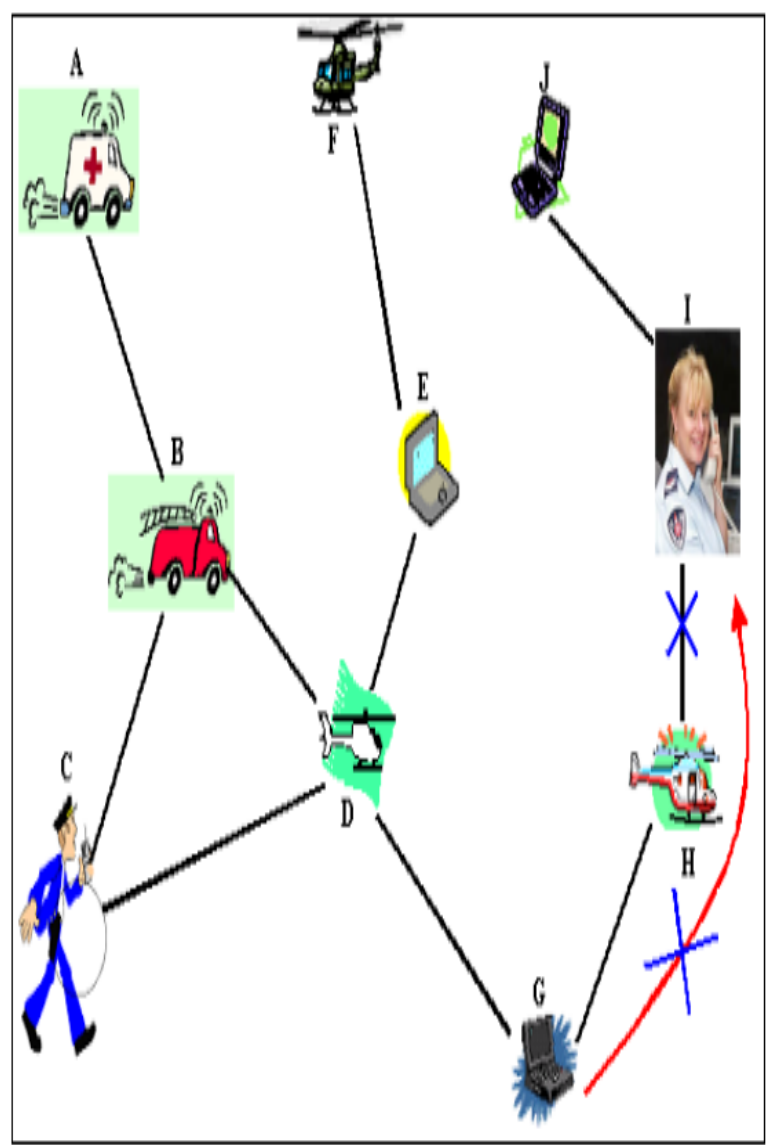

Fig. 4. A Mobile Adhoc Network to Solve Rescue Operation Challenges (Veerasamy, 2016; Anibrika et al., 2020)

\section{SIMULATION STUDY}

\subsection{Introduction}

The use of real life measurements is certainly costly (Chiang et al., 1997). Therefore, the best approach to adopt is a simulated environment.

\subsection{Mobility}

Nodal mobility are explained by the models known as Mobility Models. Also, simulating mobility model is intended to reveal the discrete nature of the routing algorithm. It shows how well the algorithm can perform when subjected to certain conditions. It can also be used for inter-group communication during the simulation stage (Arun and Shweta, 2016; Anibrika et al., 2020; V. Davies; Tan et al., 2002).

\subsection{A Scenario of the Simulation Algorithm}

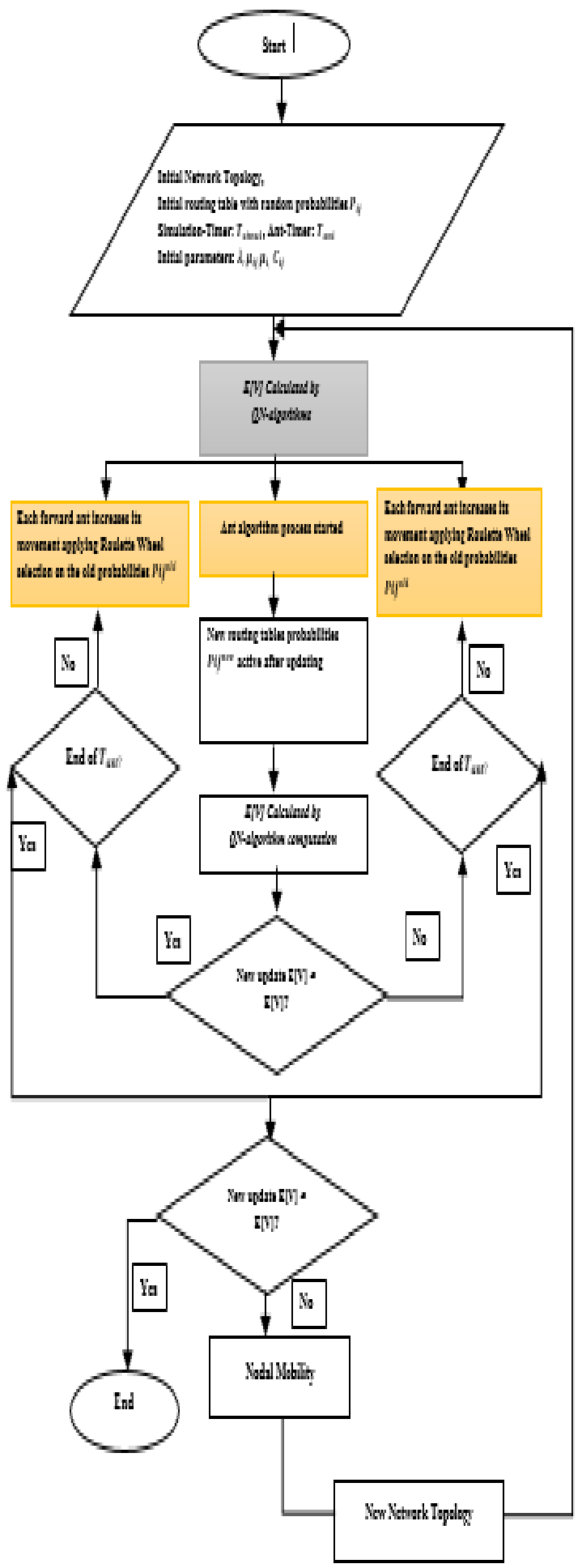

Fig. 5. Flowchart of the simulation OMRA algorithm 


\subsection{Flow Chart of Ant Colony Optimization}

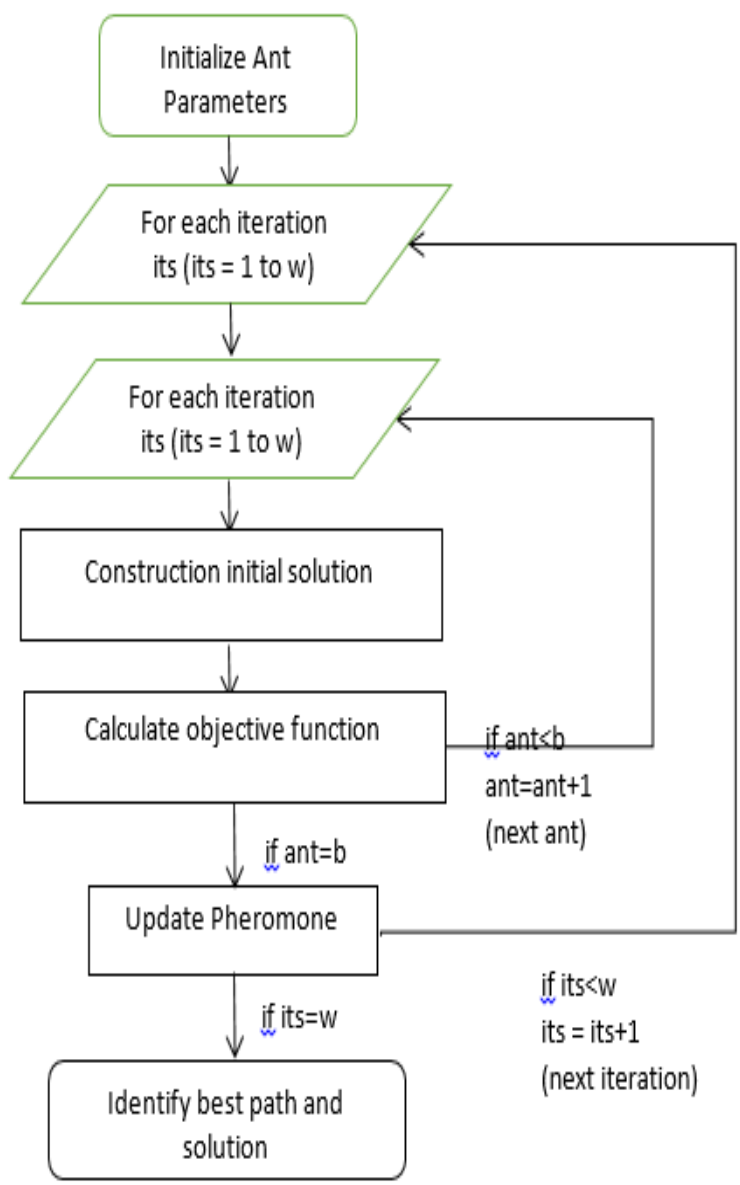

Fig.6. ACO Flowchart

\subsection{The AODV Framework}

Routers within the network move indiscriminately and connect to themselves subjectively. The AODV protocol was developed with the following objectives (Arun and Shweta, 2016):

i. To broadcast routes discovery packets.

ii. To differentiate among local node connectivity management.

iii. General topology maintenance

iv. To circulate updates in the routing table in a localized fashion to the neighboring nodes.

Each node in AODV is able to maintain two major counters:

i. A Broadcast-ID: It is increased at the source node by generating a new Route Request (RREQ) message.

ii. A Sequence Number: It is a counter which is increased sequentially so as to maintain current updates.

i. The nodes maintain localized information.

The two major mechanisms that characterize AODV routing protocol are; route discovery, route maintenance. Find further explanation in the diagram below.

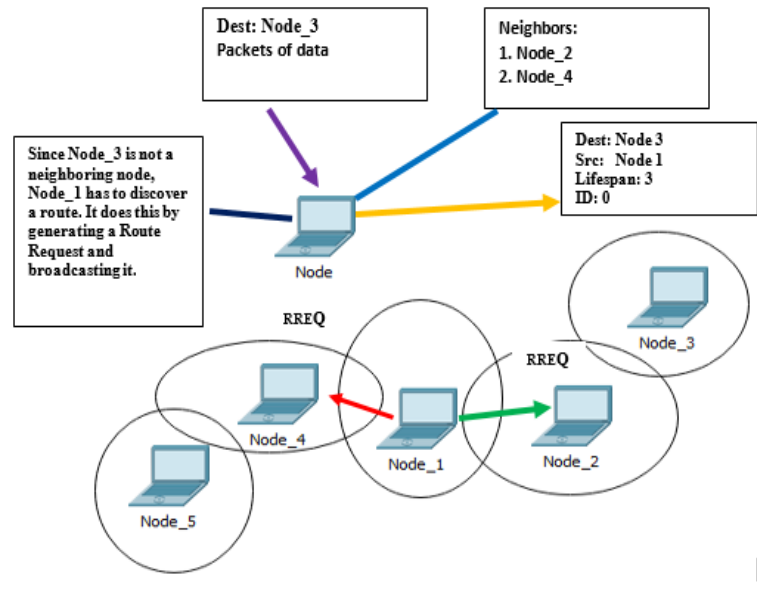

Fig. 7. RREQ Message to Destination Node. (Anibrika et al., 2020) The Algorithm for AODV

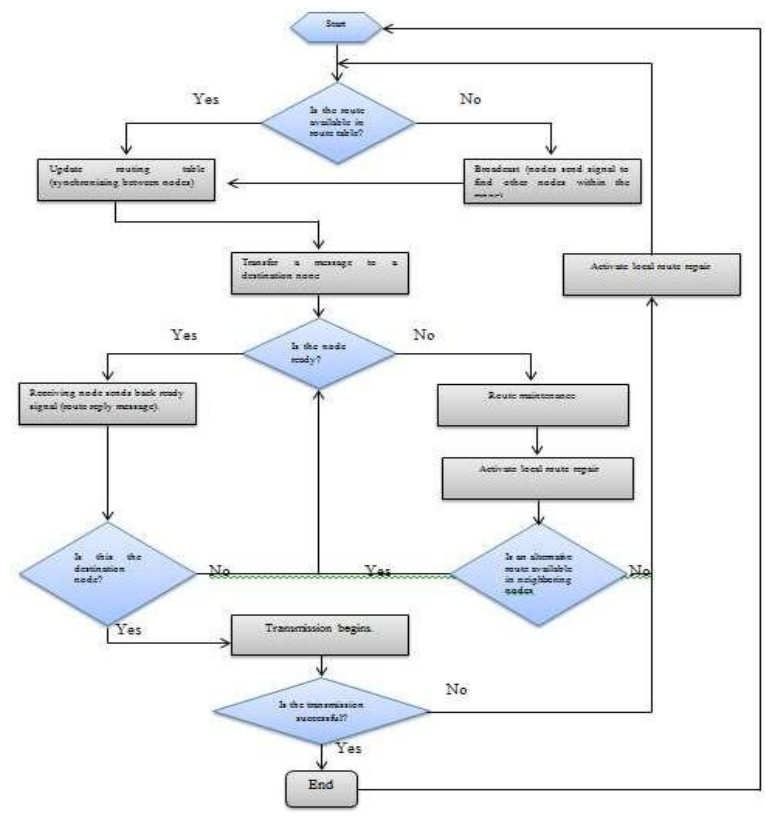

Fig.8. AODV Flowchart

\subsection{The Proposed Algorithm (Optimized Multicast Routing Algorithm)}

It has the following properties:

i.It is proactive

ii.The propagation technique employed are; Anycast, Unicast and Multicast.

iii.The proposed algorithm can create routing table entries in each node, Network Table (NT), Optimum Routes Table (ORT). It saves all optimum routes to each node in the network.

i. The algorithm manages route repair and maintenance.

ii. Two hops a registered from the new node.

iii. It will initiate a route discovery by building and saving routes the nodes.

iv. The nodes acquire information from any route that passes through. The diagram below gives further explanation, Fig. 9. 


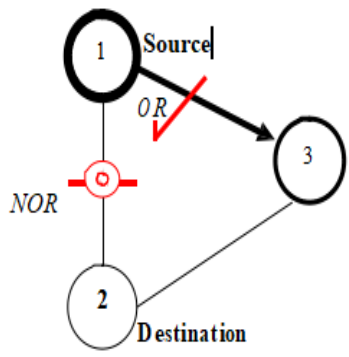

$\checkmark$ OR $=$ Optimum Route $-O-N O R=$ Non Optimum Route

Fig. 9. Optimum and No Route between 3 Neighbours

\subsection{Local Environment Setup}

Several discrete event simulation systems have been proposed for MANET, namely: NS2, Opnet, Simulink, Qualnet, OMNet++, NetSim, etc. Upon evaluation of the simulators above based on the objectives of this paper, Mathlab was used. Mathlab describes an environment for multi paradigm and numerical computing environment with programming languages integrated. It allows for the manipulation of matrices, plotting functions, algorithms, implementation and creation of user interfaces. Mathlab is written in $\mathrm{C} / \mathrm{C}++$ and Java.

\subsubsection{Network Configuration}

The topology of MANET is plotted on to a fixed graph with nodes $(N)$ and links $(M)$. The links could be described as pipes, indicating two parameters. These parameters are bandwidth in bits per secs and a delay in transmission in seconds. In this simulation, every node is able to store and forward routing information holds a buffer space (queue) as described in Fig.10 below.

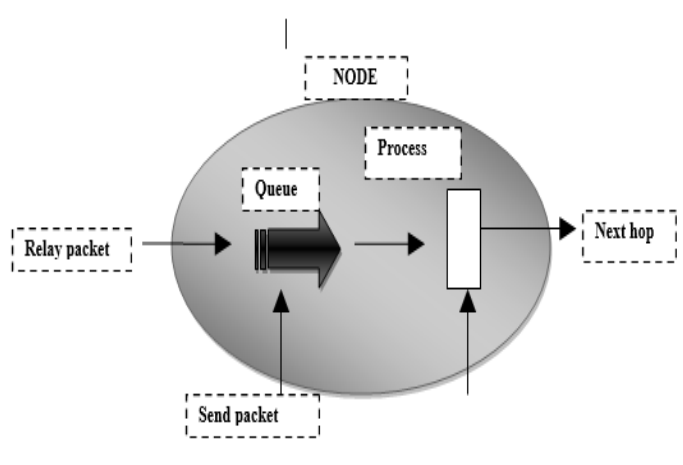

Fig.10. The Node Model

Packets are put in a queue and transmitted on First-In-FirstOut (FIFO) procedure.

\subsubsection{Routing Table Initialization}

The probability value $P_{i n}$ expressing the quality of selecting $n$ as the next node. It is influenced by the constraint:

$$
\sum_{n \in N_{k}} P_{\text {in }}=1 \in\left[1, N_{k}\right], N_{k}=\{\text { neighbors }(k)\} ;
$$

\subsubsection{Parameter Initialization}

The simulation process must begin by defining $\lambda$ denoting the arrival time. The service rate $\mu i j$ also denotes the bandwidth of two nodes, $i$ and $j$ within the network. These parameters ( $i$ and $j$ ) are used to determine the average packets serviced in each unit of time.

\subsubsection{Timer Initialization}

The timers are initialized at beginning of the simulation. The ant timer $T_{\text {ant }}$ denotes the termination condition for ant algorithm. The simulation timer $T_{\text {simul }}$ denotes time the ant algorithm will be repeated each $T_{\text {ant }}$. For instance, if $T_{\text {simul }}$ $=180 \mathrm{secs}$. and $T_{\text {ant }}=30 \mathrm{secs}$., the ant algorithm 6 repetitions on 6 separate topologies.

\subsubsection{Network Parameters and Assumptions}

i. End-to-End Delay: It is the total estimated time taken by a data packet during packet transmission destination. Mathematically calculated as: E2E Delay $=$

ii. $\Sigma=$

$$
\begin{aligned}
& \text { (Arrival time }- \text { send time }) / \\
& \Sigma \text { (Number of connections) }
\end{aligned}
$$

iii. Packet Delivery Ratio: The acute failure of the router to transfer and deliver packets of data because of loss of connections. Calculated as: $P D R=$ Sum of no. of packets recieved/ Sum of no. of packets sent

iv. Throughput: It is the ratio of output with respect to input signals, due to different load of users sharing network resources, known as bit rate.

v. Simulation Model A simulation area $1000 \times 800 \mathrm{~m} 2$ is set for this simulation based on the random waypoint model. Find below in Table 1, details of the simulation parameters and the corresponding values. The diagram Table 1, outlines the

\begin{tabular}{|c|c|}
\hline Parameter & Value \\
\hline Number of nodes & $100,200,300 \ldots \ldots 1000$ \\
\hline i. $\quad$ Rate of Arrival & $150 \mathrm{Kbps}$ \\
\hline $\begin{array}{cc}\text { ii. } & \text { Range of } \\
\text { Transmission }\end{array}$ & $250 \mathrm{~m}$ \\
\hline $\begin{array}{c}\text { Velocity and } \\
\text { Direction }\end{array}$ & $10 \mathrm{~m} / \mathrm{sec} \& 45$ degree \\
\hline iv. $\quad$ Size of Packet & 64 byte \\
\hline $\begin{array}{c}\text { Pause time } \\
(\text { Ant time })\end{array}$ & $10,20, \ldots, 100 \mathrm{sec}$ \\
\hline $\begin{array}{cc}\text { vi. } & \text { Time of } \\
\text { Simulation }\end{array}$ & $180 \mathrm{sec}$. \\
\hline $\begin{array}{l}\text { bandwidth } \\
\text { Link }\end{array}$ & $1 \mathrm{Mbps}$ \\
\hline $\begin{array}{cc}\text { viii. } \quad \begin{array}{c}\text { area of } \\
\text { Simulation }\end{array}\end{array}$ & $1000 \mathrm{~m} \times 800 \mathrm{~m}^{2}$ \\
\hline ix. $\quad$ Model & RWM \\
\hline $\begin{array}{ll}x . & \text { Protocols }\end{array}$ & AODV, OMRA \\
\hline
\end{tabular}
configurations and its parameters for the simulation.

Table 1. Simulation Parameters

\subsection{Mobility Modelling for MANET}

This paper explores the following mobility models for the simulation (V. Davies, 2002): 
i. Random Way Point:

A node selects a random position with the parameters $(x, y)$. This point becomes the destination point. Velocity (V) is chosen by computing the distributed range of parameters [minspeed, maxspeed]. This process is repeated based on the destination and speed.

ii. Random Walk

It enables the nodes to freely move about selecting a speed and a direction value in constant time intervals $(\Delta t)$.

\section{iii. Random Direction}

The nodes choose an arbitrary direction within the specific range $[0,2 \Pi]$. Again, the nodes' velocity is chosen consistently from the specified range [minspeed, maxspeed].

\section{EXPERIMENTAL RESULTS AND ANALYSIS}

These graphs are labeled and interpreted.

The newly proposed ant algorithm was simulated using the three mobility models.

\section{i. Graph of three Mobility Models}

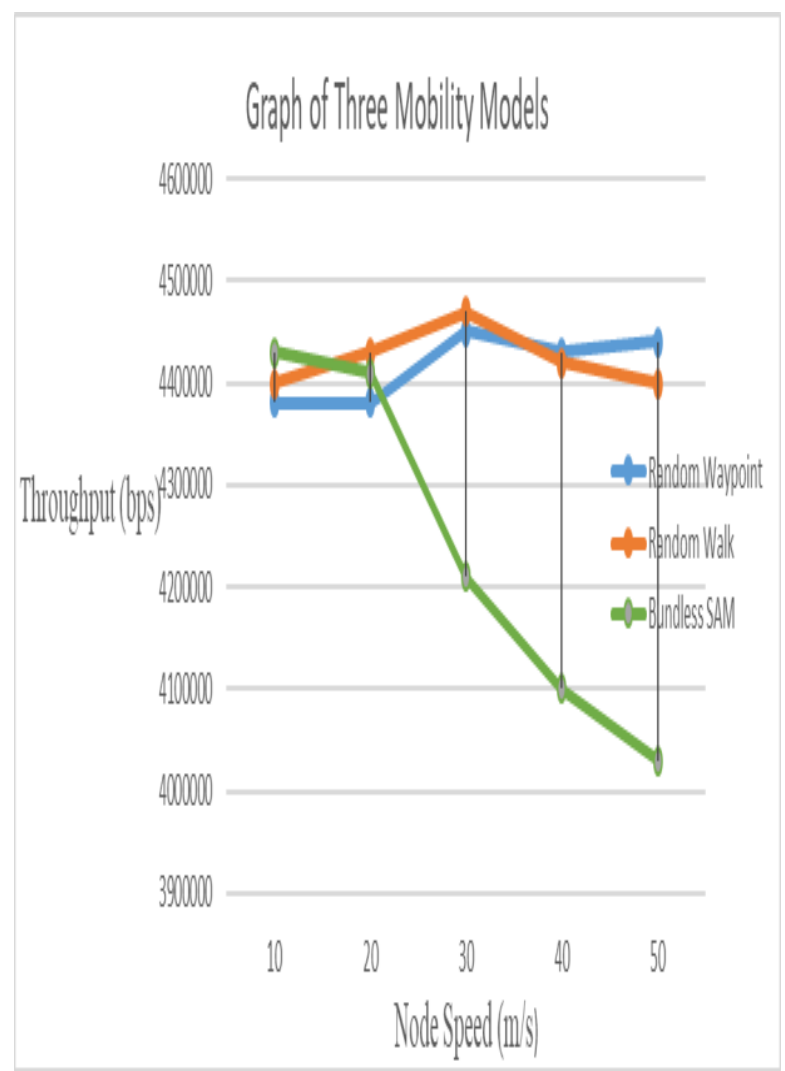

Fig. 11. Simulation of the Mobility Models

In Fig.11 the results for Throughput are presented. The nodes' speed vary from $10 \mathrm{~m} / \mathrm{s}$ to $50 \mathrm{~m} / \mathrm{s}$. From fig. 11, it is realized that, the performance of OMRA with respect to Throughput under Random Waypoint and Boundless models have certain similarities with very little differences. However, an increment in the speed of the node, affects the Throughput value as it is seen to have been steady. Random Walk however, demonstrates a gradual decline in the throughput value possibly due pace at which the nodes were communicating. In terms of Boundless Simulation Area Mobility, as the speed of the node increases a considerable drop in the throughput is observed. ii. End to End Delay for Mobility Models

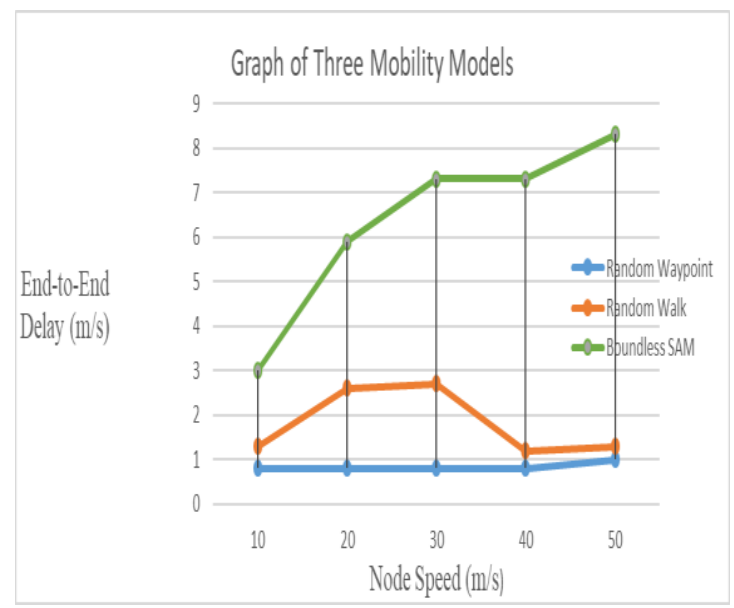

Fig. 12. OMRA with End-to-End Delay

The Packet Delay values generated by OMRA protocol with the three simulation mobility models under consideration for the purposes of this research work is demonstrated in fig. 12. When the OMRA protocol was simulated with End-to-End Delay $(1 \mathrm{~m} / \mathrm{s})$, it exhibited the least delay value. Whereas, under Random Walk Mobility model $(1.3 \mathrm{~m} / \mathrm{s})$, the delay is greater compared to Random Waypoint. The value is better than the one obtained for Boundless Simulation Area Mobility model $(3 \mathrm{~m} / \mathrm{s})$, exhibiting high values of End-to-End Delay as there is an increment in the speed of the nodes.

iii. Packet Delivery Ration in OMRA

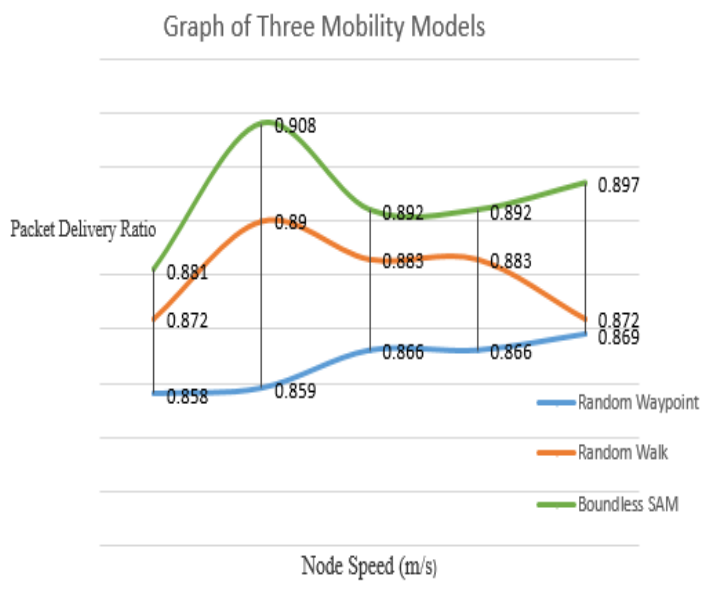

Fig. 13. The Packet Delivery Ration in OMRA with Mobility Models.

Fig.13, presents Packet Delivery Ratio graph for OMRA algorithm. It is realized that, the Boundless Simulation Area Mobility Model $(0.98 \mathrm{sec})$ is improved providing a better performance value with regards to the Packet Delivery Ratio when compared to the remaining models. This improvement, however comes at the cost of low Throughput and high Endto-End Delay. With regards to the remaining two models, Random Walk (0.89 secs) provided better and enhanced Packet Delivery Ratio which is better than the Random Waypoint value (0.859). Also, the performance of Random Waypoint Mobility model concerning Packet Delivery Ratio greatly improved due to gradual increase in speed node but the Random Walk Mobility exhibited unreliable and different values for Packet Delivery Ratio with regards to speed variations. 
iv. Throughput for Mobility Models with Time

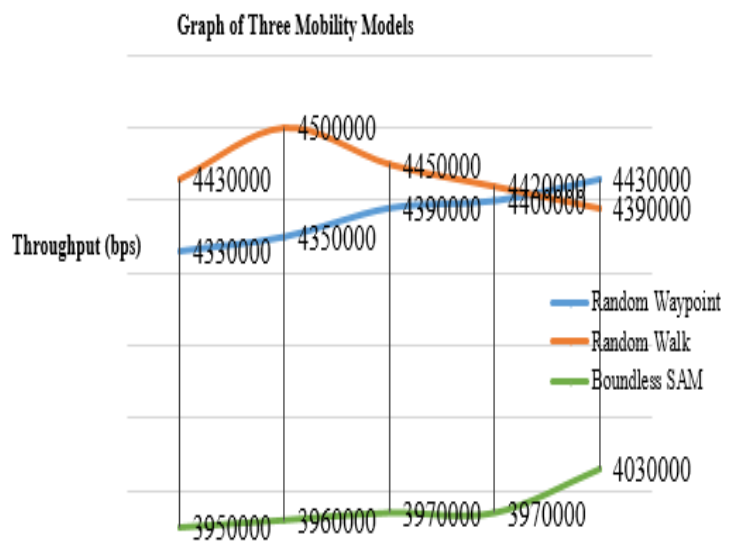

Time (Seconds)

OMRA's performance with the three mobility models over a certain time produced that were obtained for node speed of $50 \mathrm{~m} / \mathrm{s}$ (50 meters per second) and Mean Delay is demonstrated in fig. 14,15 and 16 respectively.

In fig. 14 above, it is evident that, the Random Waypoint (4330000 bps) and Random Walk (4430000) are relatively similar with specific reference to Throughput whereas the Throughput for Random Waypoint is comparatively stable. The Throughput for Random Walk portrays quite a steady decline for a time period. However, the End-to-End Delay, as explained in fig. 15 below, is the lowest in terms of Random Waypoint, but the highest for Boundless Simulation Area (3950000), especially when it is being used for the simulation.

From the results shown in fig. 16, random waypoint $(0.8$ secs $)$ seems to outperform the other two mobility models under consideration. It rather it exhibited low throughput and high end-to-end delay. The Random walk value of 1 secs provided better Packet Delivery Ratio than random waypoint mobility model.

\section{v. $\quad$ End to End Delay for Mobility Models}

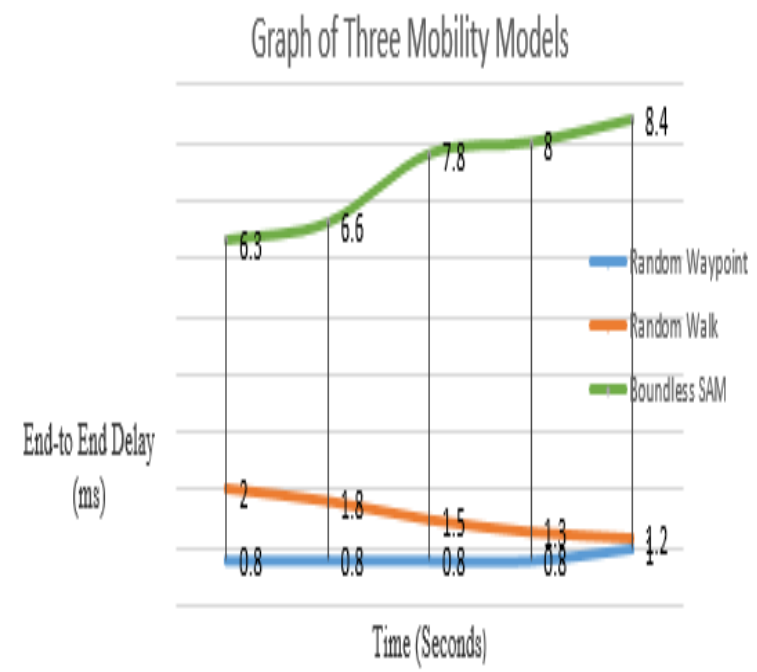

Fig. 15. End to End Delay for Mobility Model with Time vi. Packet Delivery Ratio for Mobility Models with Time Graph of Three Mobility Models

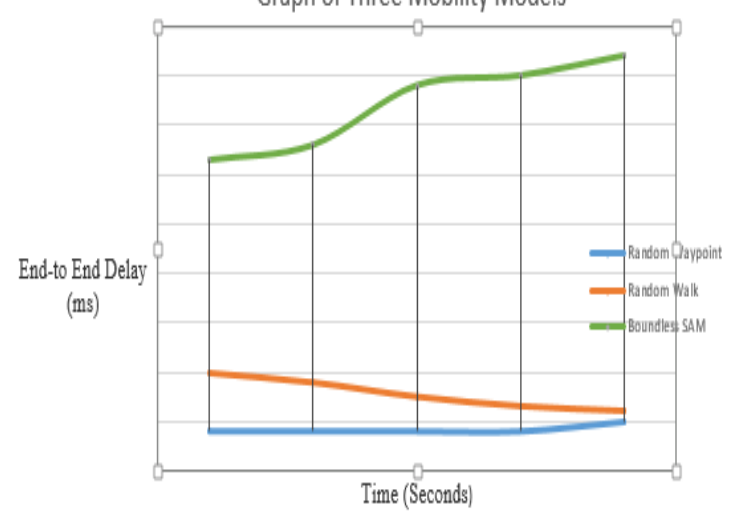

Fig. 16. Packet Delivery Ratio for Mobility Models

\subsection{Conclusions/Recommendations}

The three mobility models that is Random Waypoint Model, Random Walk Model and Boundless Simulation Area Mobility Model and their impact was demonstrated through simulation using

Throughput, End-to-End Delay, Packet Delivery Ratio and Mean Delay are the major quantitative metrics that characterize network latency within a network. These network metrics have been examined through simulation.

The output of the simulation results showed clearly that each mobility model was able to outperform the other two in respect of one of the quantitative metrics mentioned above. By considering the three parameters as a whole, it was clear that, the performance of Boundless Simulation Area seems not to perform better than the remaining two mobility models. This is because, it provided a better packet delivery, at the expense of lower Throughput and higher End-to-End Delay and so far as Random Walk and Random Waypoint is considered, OMRA especially with regards to Random Waypoint Mobility is able to provide better Throughput with low End-to-End Delay.

However, concerning Packet Delivery Ratio, the Random Walk is able to outperform that of the Random Waypoint Mobility Model .It is also evident from the simulation results that OMRA's performance under the various quantitative metrics differ from one type of mobility model to another.

\section{REFERENCES}

[1].Perkins and Bhagwat, 1994] Charles E. Perkins and Pravin Bhagwat, Highly dynamic destination-sequenced distance-vector routing (DSDV) for mobile computers. In ACM SIGCOMM'94 Conference on Communications Architectures, Protocols and Applications, pp. 234-244, 1994.

[2].Royer at al., 2001] Elizabeth M. Royer, P. M. MelliarSmith, Louise E. Moser. An Analysis of the Optimum Node Density for Ad hoc Mobile Networks. Proceedings of the IEEE International Conference on Communications, Helsinki, Finland, 2001.

[3].Patel, R.; Kamboj, P. Investigation of Network Simulation Tools and Comparison Study: NS3 vs NS2. J. Netw. Commun. Emerg. Technol. JNCET 2015, 5, 137-142

[4].Jha RK, Limkar SV, Dr. Dalal UD, A Performance Comparison of Routing Protocols (DSR and TORA) for 
Security Issue in MANET (Mobile Ad Hoc Networks), International Journal of Computer Applications, Special Issue on "Mobile Ad-hoc Networks" MANETs, 2010, pp. 78-83.

[5]. Sunil Kumar, Mishra R, Krishna Kumar, Singh BM, An Inspection of Mobility Models Routine on Routing Protocols in Ad Hoc Network, International Journal of Engineering and Innovative Technology (IJEIT), 2013, 2(8), pp. 269-274.

[6].Tonk G, Kashyap I, Tyagi SS, Performance Comparison of Ad-Hoc Network Routing Protocols using NS-2, International Journal of Innovative Technology and Exploring Engineering (IJITEE), 2012 1(1), pp. 53-57.

[7].Singh P, Bhola A, Jha CK, Simulation based Behavioral Study of AODV, DSR, OLSR and TORA Routing Protocols in Manet, International Journal of Computer Applications, 2013, 67(23), pp. 23-26.

[8]. Nand P, Dr. Sharma SC, Performance Study of Broadcast based Mobile Adhoc Routing Protocols AODV, DSR and DYMO, International Journal of Security and its Applications, 2011, 5(1), pp. 53-64.

[9].Subramani A, Krishnan A, Node Mobility Tracking in Mobile Ad-Hoc Networks in their Geographical Position (Dynamic Networks), International Journal of Soft Computing and Engineering (IJSCE), ISSN: 2231-2307, 2011, 1(5), pp. 36-40.

[10]. Shukla AK, Jha CK, Sharma D, An Estimation of Routing Protocols in Mobility Models Used for Ad Hoc Networks: Simulation Study, International Conference on Advances in Computer Application (ICACA-2013), Proceedings Published in International Journal of Computer Applications (IJCA) (975-8887), pp. 21-27.
[11].Rafiq M, Manoj Kumar, Ahmed S, Comparative Analysis of Energy Efficient Routing Protocols in MANET, International Journal of Computer Applications, 2013, 82(5), pp. 1-6.

[12]. [Atsushi Iwata et al., 1999] Atsushi Iwata, Ching-Chuan Chiang, Guangyu Pei, Mario Gerla, and Tsu Wei Chen. Scalable routing strategies for ad hoc wireless networks. Tech. Rep., Department of Computer Science University of California, Los Angeles, 1999.

[13].M. Bergamo. System design specification for mobile multimedia wireless network (MMWN). Technical report, DARPA project, 1996.

[14]. [Chiang et al., 1997] Ching-Chuan Chiang, Hsiao-Kuang $\mathrm{Wu}$, Winston Liu, and Mario Gerla. Routing in clustered multihop, mobile wireless networks with fading channel. Tech. Rep., University of California at Los Angeles Computer Science Department, 1997.

[15]. [Chlamtac et al., 2003] Chlamtac, M. Conti and J. J.-N. Liu. Mobile ad hoc networking: imperatives and challenges. Ad Hoc Networks, Vol. (1), pages 13-64, 2003.

[16].V. Davies. Evaluating mobility models within an ad hoc network. Master's thesis, Colorado School of Mines, 2000.

[17].D.S.Tan, S. Zhou, J. Ho, J.S. Mehta, H. Tanabe. Design and Evaluation of an Individually Simulated Mobility Model in Wireless Ad Hoc Networks. Communication Networks and Distributed Systems Modeling and Simulation Conference, San Antonio, 2002.

[18].M. Dorigo, M. Birattari and T. Stutzle, "Ant colony optimization," in IEEE Computational Intelligence Magazine, vol. 1, no. 4, pp. 28-39, Nov. 2006, DOI: 10.1109/MCI.2006.329691 